\title{
HARMONIC FUNCTIONS FOR A CLASS OF MARKOV CHAINS
}

\author{
H. $\mathrm{COHN}$ \\ (Received 15 June 1978; revised 16 February 1979) \\ Communicated by R. L. Tweedie
}

\begin{abstract}
A class of Markov chains is considered for which a certain property of the tail events makes bounded harmonic functions obtainable from bounded space-time harmonic functions. Applications to almost surely convergent Markov chains are given and, in particular, a representation of Martin-Doob-Hunt type is derived for all bounded harmonic functions of a finite mean supercritical branching process.
\end{abstract}

1980 Mathematics subject classification (Amer. Math. Soc.): $60 \mathrm{~J} 05$.

\section{Introduction}

Let $S$ be a countable set of integers, $N=\{0,1, \ldots\}$ and $\Omega=S^{N}$. Define the variables $\left\{X_{n}(\omega): n \geqslant 0\right\}$ on $\Omega$ by $X_{n}(\omega)=\omega_{n}$ where $\omega=\left(\omega_{0}, \omega_{1}, \ldots, \omega_{n}, \ldots\right)$ and let $\mathscr{F}$ be the $\sigma$-field generated by the variables $\left\{X_{n}(\omega): n \geqslant 0\right\}$. Suppose that $\pi^{(0)}=\left(\pi_{i}^{(0)}: i \in S\right)$ is an initial probability vector whose components are strictly positive. Write $P=(P(i, j): i, j \in S)$ for the transition probability matrix of the chain. The pair $\left(\pi^{(0)}, P\right)$ determines a probability measure $P$ on $\mathscr{F}$ and a temporally homogeneous Markov chain $\left\{X_{n}(\omega): n \geqslant 0\right\}$ on $(\Omega, \mathscr{F}, P)$ such that $\pi_{i}^{(0)}=P\left(X_{0}=i\right)$ and $P\left(X_{n}=j \mid X_{n-1}=i\right)=P(i, j)$ whenever $P\left(X_{n-1}=i\right)>0$. Apart from the probability measure $P$ defined above we shall consider the probability measures $\left\{P_{i}\right\}$ with $i \in S$ where $P_{i}$ stands for the probability measure of a chain assuming the transition matrix $P$ and the Dirac measure $\delta(i)$ for the initial probability distribution. An expectation or a distribution function of a random variable will be denoted by $E_{i}$ and $F_{i}$ respectively, when taken with respect to $P_{i}$. A shift function $T: \Omega \rightarrow \Omega$ is defined by setting $T\left(\omega_{0}, \omega_{1}, \ldots\right)=\left(\omega_{1}, \omega_{2}, \ldots\right)$. We shall write $T \Lambda=\{T \omega: \omega \in \Lambda\}$, $\mathrm{T}^{-1} \Lambda=\{\omega: T \omega \in \Lambda\}$ and $T^{0} \Lambda=\Lambda$. A set $I$ in $\mathscr{F}$ is said to be invariant if $T^{-1} I=I$. 
The class of all invariant sets, denoted by $\mathscr{I}$, is a $\sigma$-field, called the invariant $\sigma$-field. Denote by $\mathscr{F}_{n}^{\infty}$ the $\sigma$-field generated by $X_{n}, X_{n+1}, \ldots$ and by $\mathscr{T}=\bigcap_{n=0}^{\infty} \mathscr{F}_{n}^{\infty}$ the tail $\sigma$-field of the chain. We shall say that $A=B$ almost surely if $P(A \Delta B)=0$ and $\lim _{n \rightarrow \infty}\left\{X_{n} \in A_{n}\right\}=\Lambda$ almost surely if

$$
P\left(\Lambda \Delta \lim _{n \rightarrow \infty} \inf \left\{X_{n} \in A_{n}\right\}=P\left(\Lambda \Delta \lim _{n \rightarrow \infty} \sup \left\{X_{n} \in A_{n}\right\}=0,\right.\right.
$$

where $A \Delta B$ stands for the symmetric difference of $A$ and $B$. Write $\pi_{i}^{(n)}=P\left(X_{n}=i\right)$ and let $S_{n}=\left\{j: \pi_{j}^{(n)}>0\right\}$. It can be easily checked that $S_{0} \supseteq S_{1} \supseteq \ldots$. We shall suppose that $\left\{X_{n}(\omega): n \geqslant 0\right\}$ is a properly homogeneous chain, that is, that $P\left(\lim \sup X_{n} \in\left\{S_{n}-S_{n+1}\right\}\right)=0$, see Cohn (1979). Let $R$ be the set of all real $\lim _{n \rightarrow \infty}$ numbers and $R_{+}$the set of nonnegative numbers. A function $h: S \rightarrow R_{+}$will be said to be harmonic if

$$
h(i)=\sum_{j \in S} P(i, j) h(j) \text { for all } i \in S
$$

and $g: N \times S \rightarrow R_{+}$will be said to be space-time harmonic if

$$
g(n, i)=\sum_{j \in S} P(i, j) g(n+1, j) \quad \text { for all } i \in S \text { and } n \in N .
$$

In abbreviated form (1) will read $f=P f$ and (2) $g_{n}=P g_{n+1}$. It is well known that to each harmonic function $f$ can be attached a martingale $\left\{f\left(X_{n}\right): n \geqslant 0\right\}$ and it is of interest to identify classes of harmonic functions of a chain. The identification of the harmonic functions is the main object of the Martin boundary theory and, roughly speaking, this is done by investigating the asymptotic behaviour of the Martin boundary kernel $K(i, j)$ defined as

$$
K(i, j)=\frac{G(i, j)}{G_{\pi}(j)},
$$

where $G(i, j)=\sum_{n=0} P^{n}(i, j)$ is the Green function of the chain, $P^{0}$ the identity matrix and $G_{\pi}(j)=\sum_{i \in S} \pi_{i}^{(0)} G(i, j)$ (see, for example, Revuz (1975), Chapter 7). Except for a few cases, the Martin boundary kernel is a very complicated expression and its handling seems to be a formidable task. Such a task was successfully carried out, under certain conditions, for the finite mean branching processes by Dubuc (1970), but seems hopeless in many other cases.

The identification of the space-time harmonic functions is somewhat easier, since the Green function of a space-time chain takes the simpler form

$$
G((m, i):(n, j))=P^{n-m}(i, j), \quad n>m, \quad i, j \in S,
$$

and there are several examples known in the literature when the space-time harmonic functions were actually identified. 
The aim of this paper is to point out a class of Markov chains for which classes of harmonic functions can be identified by means of space-time harmonic functions. The basic idea, suggested by the branching processes case, is the following: if we have a space-time harmonic function $g$, we can 'extend' it by defining $P g_{0}=g_{-1}$, $P g_{-1}=g_{-2}$, and so on. Suppose that $h(i)=\sum_{n=-\infty}^{\infty} g(n, i)<\infty$ for all $i \in S$. Then we can easily check that $h=P h$ and hence $h$ is an harmonic function. In general $\sum_{n=-\infty}^{\infty} g(n, i)$ need not be finite and this construction does not always lead us to an harmonic function. However, we shall define a class of Markov chains containing some cases of interest, for which this purely analytical construction turns out to have some probabilistic meaning and classes of harmonic functions will be identified in this way.

The main probabilistic assumption is expressed by the following.

Condrion (A). There exists a set $\Lambda_{0} \in \mathscr{T}$ with $P\left(\Lambda_{0}\right)>0$ such that

(a) For any $m$ and $n$ with $m \neq n, P\left(T^{m} \Lambda_{0} \cap T^{n} \Lambda_{0}\right)=0$,

(b) $P\left(\bigcup_{n=-\infty}^{\infty} T^{n} \Lambda_{0}\right)=1$.

LEMMA 1. Suppose that Condition (A) holds. Then a set $I$ is invariant if and only if it can be represented as

$$
I=\bigcup_{n=-\infty}^{\infty} T^{n} \Lambda \text { almost surely }
$$

for some $\Lambda \in \mathscr{T}$, with $\Lambda \subseteq \Lambda_{\mathbf{0}}$.

Proof. Suppose that $I$ is an invariant set and consider the sets $I_{n}=I \cap T^{n} \Lambda_{0}$ with $n \in Z$. Then according to Proposition 1 of Cohn (1979), $I_{n}=T^{n}\left(I \cap \Lambda_{0}\right)$ and $\mathrm{U}_{n=-\infty}^{\infty} I_{n}=I$ almost surely. Reciprocally, $\bigcup_{n=-\infty}^{\infty} T^{n} \Lambda$ is an invariant set for any $\Lambda \in \mathscr{T}$ and the lemma follows.

We shall further agree to modify the definition of the space-time harmonic functions $g$ to make it contain the negative values of $n$ which were defined above, that is, a sequence $\left\{g_{n}: n \in Z\right\}$ will henceforth be said to be space-time harmonic if for any $n \in Z, g_{n}=P g_{n+1}, Z$ being the set of all integers.

Theorem 1. Suppose that Condition $(A)$ holds. Then any bounded harmonic function $h$ can be represented as

$$
h=\sum_{n=-\infty}^{\infty} g_{n}
$$

where $\left\{g_{n}: n \in Z\right\}$ is a space-time harmonic function. 
Proof. According to Blackwell (1955), Theorem 2, any bounded harmonic function $h$ is expressible as $h(i)=E\left(f \mid X_{0}=i\right)$ where $f$ is an invariant function. Furthermore, any invariant function $f$ is the limit of increasing simple invariant functions

$$
f_{m}=\sum_{k=1}^{m} a_{k}^{(m)} 1_{A_{x}^{(m)}}
$$

where $A_{1}^{(m)}, \ldots, A_{m}^{(m)}$ are invariant sets, $a_{1}^{(m)}, \ldots, a_{m}^{(m)}$ are positive numbers and $1_{\Lambda}$ is the indicator of the set $A$.

Consider now the conditional expectation

$$
E\left(f_{m} \mid X_{0}=i\right)=\sum_{k=1}^{m} a_{k}^{(m)} P_{i}\left(A_{k}^{(m)}\right) .
$$

By Lemma 1 there exists a set $\Lambda_{k}^{(m)}$ with $\Lambda_{k}^{(m)} \subseteq \Lambda_{0}$ such that

$$
A_{k}^{(m)}=\bigcup_{n=-\infty}^{\infty} T^{n} \Lambda_{k}^{(m)}
$$

Since $P\left(T^{l} \Lambda_{k}^{(m)} \cap T^{n} \Lambda_{k}^{(m)}\right)=0$ for $l \neq n$ we get

$$
P\left(A_{k}^{(m)} \mid X_{0}=i\right)=\sum_{n=-\infty}^{\infty} P\left(T^{n} \Lambda_{k}^{(m)} \mid X_{0}=i\right) .
$$

(3) and (4) together imply

$$
\begin{aligned}
E\left(f_{m} \mid X_{0}=i\right) & =\sum_{k=1}^{m} a_{k}^{(m)} \sum_{n=-\infty}^{\infty} P\left(T^{n} \Lambda_{k}^{(m)} \mid X_{0}=i\right) \\
& =\sum_{n=-\infty}^{\infty} \sum_{k=1}^{m} a_{k}^{(m)} P\left(T^{n} \Lambda_{k}^{(m)} \mid X_{0}=i\right) .
\end{aligned}
$$

Since for any $k$ and $m,\left\{P\left(T^{n} \Lambda_{k}^{(m)} \mid X_{0}=i\right): n \in Z\right\}$ is easily checked to be a spacetime harmonic function, the same will hold for the $\left\{g_{n}^{(m)}(i): n \in Z\right\}$ for any $m \in N$ where $g_{n}^{(m)}(i)=\sum_{k=1}^{m} a_{k}^{(m)} P\left(T^{n} \Lambda_{k}^{(m)} \mid X_{0}=i\right)$. It is easy to see that $\left\{g_{n}^{(m)}(i)\right\}$ is increasing with respect to $m$ for any $i \in S$ and hence

$$
\lim _{m \rightarrow \infty} g_{n}^{(m)}(i)=g_{n}(i)
$$

exists for any $i \in S$.

Therefore

$$
h=\sum_{n=-\infty}^{\infty} g_{n}
$$

and since $g_{n}=\lim _{m \rightarrow \infty} g_{n}^{(m)}=\lim _{m \rightarrow \infty} P g_{n+1}^{(m)}=P \lim _{m \rightarrow \infty} g_{n+1}^{(m)}=P g_{n+1}$, it follows that $\left\{g_{n}: n \in Z\right\}$ is space-time harmonic and the proof is complete. 


\section{Harmonic functions for conrergent sequences}

Suppose that $\left\{X_{n}(\omega): n \geqslant 0\right\}$ is a Markov chain with the property that there exist norming constants $\left\{a_{n}\right\}$ and $\left\{b_{n}\right\}$ with $a_{n}>0$ such that $\lim _{n \rightarrow \infty} a_{n}\left(X_{n}+b_{n}\right)=V$ almost surely exists, is proper and nondegenerate. It was proved in Cohn (1977a), Theorem 4, that $\lim _{n \rightarrow \infty} a_{n+1} / a_{n}=\alpha$ and $\lim _{n \rightarrow \infty} a_{n+1}\left(b_{n+1}-b_{n}\right)=\beta$ exist, are finite and $\alpha>0$. We shall write $F$ for the distribution function of $V$. In the course of the proof of Theorem 4 of Cohn (1979) it was shown that if $\gamma$ is a jump point for $F$, then

$$
\begin{gathered}
\gamma \alpha^{k}+\beta\left(\alpha^{k}-1\right)(\alpha-1)^{-1}, \quad k=0,1, \ldots, \\
(\gamma-\beta) \alpha^{-k}-\beta \alpha^{-1}\left(\alpha^{-k+1}-1\right)\left(\alpha^{-1}-1\right)^{-1}, \quad k=1,2, \ldots
\end{gathered}
$$

are also jump points for $F$ and

(5) $T^{k}\{V=\gamma\}=\left\{V=\gamma \alpha^{k}+\beta\left(\alpha^{k}-1\right)(\alpha-1)^{-1}\right\}$ almost surely,

$$
T^{-k}\{V=\gamma\}=\left\{V=(\gamma-\beta) \alpha^{-k}-\beta \alpha^{-1}\left(\alpha^{-k+1}-1\right)\left(\alpha^{-1}-1\right)^{-1}\right\} \text { almost surely, }
$$

for $k=0,1, \ldots$.

If $F$ is absolutely continuous in an interval $(a, b)$ we shall denote by $f(x, i)$ (for $x \in(a, b))$ the density of $V$ with respect to the probability measure $P_{i}$ and write $\lambda_{0}=\beta /(1-\alpha)$.

Consider now the functions

$$
h(x, i)=\sum_{n=-\infty}^{\infty} P_{i}\left(V=\lambda_{0}+\alpha^{n}\left(x-\lambda_{0}\right)\right)
$$

where $x$ is a jump point for $F$ and

$$
h(x, i)=\sum_{n=-\infty}^{\infty} \alpha^{n} f\left(\lambda_{0}+\alpha^{n}\left(x-\lambda_{0}\right), i\right)
$$

for $x \in(a, b)$.

We shall denote by $\sigma(V)$ the $\sigma$-algebra generated by the random variable $V$ and write $Y_{n}=a_{n}\left(X_{n}+b_{n}\right), n \geqslant 0$.

THEOREM 2. Suppose that $\left\{Y_{n}: n \geqslant 0\right\}$ converges almost surely to a proper and nondegenerate random variable $V, \alpha>1$, and that

$$
\ldots<x_{-k}<x_{-k+1}<\ldots<x_{0}<\ldots<x_{k}<x_{k+1}<\ldots
$$

with $\lim _{n \rightarrow \infty} x_{n}=\infty, \lim _{n \rightarrow-\infty} x_{n}=-\infty$ are jump points for $F$. Assume further that $F$ is absolutely continuous in any interval $\left(x_{n}, x_{n+1}\right)$ with $n \in Z$. Then

(a) $\{h(x, i)\}$ are harmonic functions, except perhaps for $x \in N, N$ being a set of Lebesgue measure 0 with $N \subset R-\left\{x_{n}: n \in Z\right\}$. 
(b) For any bounded harmonic function $h$ of the form $h(i)=E_{i}(U)$ where $U$ is an invariant random variable measurable with respect to $\sigma(V)$, there exists a unique positive measure $\eta$ on $R$ such that

$$
h(i)=\eta\left(\lambda_{0}\right) P_{i}\left(V=\lambda_{0}\right)+\sum_{\nu=1}^{m} \eta\left(\gamma_{\nu}\right) h\left(\gamma_{\nu}, i\right)+\int_{\Gamma} h(x, i) \eta(d x),
$$

where $\lambda_{0}=\beta /(1-\alpha)$,

$$
\Gamma=\left(\lambda_{1}, \alpha \lambda_{1}+\beta\right) \cup\left\{\lambda_{0}\right\} \cup\left(\lambda_{2}, \alpha \lambda_{2}+\beta\right)-\left\{\gamma_{\nu}: \nu=1, \ldots, m\right\},
$$

$\lambda_{1}>\lambda_{0}$ and $\lambda_{2}<\lambda_{0}$.

Proof. As in the proof of Theorem 4 of Cohn (1979) it can be shown that

and

$$
T\{a<V \leqslant b\}=\{\alpha a+\beta<V \leqslant \alpha b+\beta\} \text { almost surely, }
$$

$$
T^{-1}\{a<V \leqslant b\}=\left\{\alpha^{-1}(a-\beta)<V \leqslant \alpha^{-1}(b-\beta)\right\} \text { almost surely, }
$$

for $a, b \neq 0$.

If we further notice that $\lambda_{0}$ is the fixed point of the mappings $x \rightarrow \alpha x+\beta$ and $x \rightarrow \alpha^{-1}(x-\beta)$, we write $\alpha x+\beta=\lambda_{0}+\alpha\left(x-\lambda_{0}\right), \alpha^{-1}(x-\beta)=\lambda_{0}+\alpha^{-1}\left(x-\lambda_{0}\right)$ and by iteration we get for $k \geqslant 1$

$$
T^{n}\{a<V \leqslant b\}=\left\{\lambda_{0}+\alpha^{n}\left(a-\lambda_{0}\right)<V \leqslant \lambda_{0}+\alpha^{n}\left(b-\lambda_{0}\right)\right\} \text { almost surely, }
$$

and

(8) $T^{-n}\{a<V \leqslant b\}=\left\{\lambda_{0}+\alpha^{-n}\left(a-\lambda_{0}\right)<V \leqslant \lambda_{0}+\alpha^{-n}\left(b-\lambda_{0}\right)\right\}$ almost surely.

Now it is easy to check that for $x \neq \lambda_{0},\left\{T^{n}\{x<V \leqslant \alpha x+\beta\}: n \in Z\right\}$ are disjoint sets, whereas $T^{n}\{x<V \leqslant \alpha x+\beta\}$ and $T^{n+1}\{x<V \leqslant \alpha x+\beta\}$ have a common end point for any $n \in Z$. Thus

$$
\bigcup_{n=-\infty}^{\infty} T^{n}\left\{V \in\left(\lambda_{1}, \alpha \lambda_{1}+\beta\right)\right\}=\left\{\lambda_{0}<V<\infty\right\} \text { almost surely, }
$$

and

$$
\bigcup_{n=-\infty}^{\infty} T^{n}\left\{V \in\left(\lambda_{2}, \alpha \lambda_{2}+\beta\right)\right\}=\left\{-\infty<V<\lambda_{0}\right\} \text { almost surely. }
$$

Hence $\left\{\lambda_{0}<V<\infty\right\}$ and $\left\{-\infty<V<\lambda_{0}\right\}$ are either invariant sets or differ from invariant sets by null sets. As a consequence, if $\lambda_{0}$ is a jump point for $F,\left\{V=\lambda_{0}\right\}$ either is also invariant or differs from an invariant set by a null set. Another consequence of (9) and (10) is that in order to get all the harmonic functions $\{h(x, i)\}$ we can limit ourselves to the values of $x$ belonging to

$$
\Delta=\left(\lambda_{1}, \alpha \lambda_{1}+\beta\right) \cup\left\{\lambda_{0}\right\} \cup\left(\lambda_{2}, \alpha \lambda_{2}+\beta\right)
$$

since for any $x \in R$ there exists $x^{\prime}$ in $\Delta$ such that $h(x, i)=h\left(x^{\prime}, i\right)$ for all $i$. 
Now if $P(V=x)>0, h(x, i)$ is harmonic, since $h(x, i)=P_{i}\left(\bigcup_{n=-\infty}^{\infty} T^{n}\{V=x\}\right)$ and Lemma 1 and the already-mentioned Theorem 2 of Blackwell (1955) apply.

Consider now the function $G_{i}(x)=P_{i}\left(\bigcup_{n=-\infty}^{\infty} T^{n}\{V \in \Lambda \cap(-\infty, x)\}\right)$ for $x \in \Gamma$, which is also harmonic. It is easy to see that

$$
\begin{aligned}
G_{i}(x)=\sum_{n=-\infty}^{\infty}\left(F_{i}\left(\lambda_{0}+\alpha^{n}\left(x-\lambda_{0}\right)\right)\right. & \left.-F_{i}\left(\lambda_{0}+\alpha^{n}\left(\lambda_{1}-\lambda_{0}\right)\right)\right) \\
& -\sum_{n=-\infty}^{\infty} P_{i}\left\{T^{n}\left(V \in\left(\lambda_{1}, \alpha \lambda_{1}+\beta\right) \cup\left\{\lambda_{0}\right\}\right)\right\}
\end{aligned}
$$

for $x \in\left(\lambda_{1}, \alpha \lambda_{1}+\beta\right)$ and

$$
G_{i}(x)=\sum_{n=-\infty}^{\infty}\left(F_{i}\left(\lambda_{0}+\alpha^{n}\left(x-\lambda_{0}\right)\right)-F_{i}\left(\lambda_{0}+\alpha^{n}\left(\lambda_{2}-\lambda_{0}\right)\right)\right.
$$

for $x \in\left(\lambda_{2}, \alpha \lambda_{2}+\beta\right)$.

Take now $x \in(a, b)$ where $(a, b)$ is an interval in which $F$ is absolutely continuous. As we have remarked before, $G(x)=\left\{G_{i}(x)\right\}$ is harmonic and $P G(x)=G(x)$ implies that its derivative $G_{i}(x)$ is also harmonic, provided that it exists and is finite. Notice now that $G_{i}^{\prime}(x)=h(x, i)$. The proof of (a) can now be completed on invoking the Fubini theorem on differentiation (see, for example, Riesz and Nagy-Sz (1955), p. 11) which asserts that the differentiation term by term is validated at all points $x \in(a, b)$ except, maybe, for a set of Lebesgue measure 0 .

To prove (b) we first notice that there are only a finite number of values $\left\{x_{n}: n \in Z\right\}$ in $\Gamma$ since we have assumed that $\lim _{k \rightarrow-\infty} x_{k}=-\infty$ and $\lim _{k \rightarrow \infty} x_{k}=\infty$. Furthermore, because all the invariant events of the form $\bigcup_{n=-\infty}^{\infty} T^{n}\left\{V=x_{m}\right\}$ are obtained for $x_{m} \in \Gamma$ it follows that there are only a finite number of invariant sets generated by the jump points of $F$, which will be denoted by $\gamma_{1}, \ldots, \gamma_{m}$.

Let us further notice that a slightly different variant of Lemma 1 entails that any invariant event can be expressed as $\bigcup_{n=-\infty}^{\infty} T^{n} \Lambda$ with $\Lambda \subseteq\{V \in \Delta\}$. It is not difficult to see that such an event can be written as

$$
\bigcup_{n=-\infty}^{\infty} T^{n} \Lambda_{1} \cup\left\{V=\lambda_{0}\right\} \cup \bigcup_{n=-\infty}^{\infty} T^{n} \Lambda_{2}
$$

with $\Lambda_{1} \subseteq\left\{V \in\left(\lambda_{1}, \alpha \lambda_{1}+\beta\right]\right\}$ and $\Lambda_{2} \subseteq\left\{V \in\left(\lambda_{2}, \alpha \lambda_{2}+\beta\right]\right\}$. Choose now

and

$$
\Lambda_{1}(a, b)=\left\{V \in\left\{(a, b) \cap\left(\lambda_{1}, \alpha \lambda_{1}+\beta\right]\right\}\right\}
$$

$$
\Lambda_{2}(c, d)=\left\{V \in\left\{(c, d) \cap\left(\lambda_{2}, \alpha \lambda_{2}+\beta\right]\right\}\right\} \text {. }
$$

Then the class of sets $\left\{\left\{V=\lambda_{0}\right\} \cup \Lambda_{1}(a, b) \cup \Lambda_{2}(c, d)\right\}$ for $a, b, c, d \in \Delta$ generates all the invariant events in $\sigma(V)$. 
Since the representation (6) is valid for

$$
h(i)=\sum_{n=-\infty}^{\infty} P_{i}\left(T^{n} \Lambda_{1}(a, b)\right)+P_{i}\left(V=\lambda_{0}\right)+\sum_{n=-\infty}^{\infty} P_{i}\left(T^{n} \Lambda_{2}(a, b)\right)
$$

a standard approximation argument in the integration theory leads us to conclude that $h(i)=E_{i}(U)$ where $U$ is a $\sigma(V)$ measurable, bounded, invariant variable and the proof is complete.

REMARK. It is easy to see that for any $x \in\left\{x_{n}: n \in Z\right\}, h(x, i) \leqslant 1$ for all $i$. However, as a function of $(x, i), h$ is unbounded. Indeed, if we choose $x \in\left(x_{m}, x_{m+1}\right)$ for a certain $m$ and take $\varepsilon>0$, the martingale convergence theorem implies that $\lim _{n \rightarrow \infty} P\left(V \in(x-\varepsilon, x+\varepsilon) \mid X_{n}\right)=1_{\left\{D_{\in}(x-8, x+\varepsilon)\right\}}$ almost surely. It follows that there exists a sequence $\left\{i_{n}\right\}$ such that $\lim _{n \rightarrow \infty} P_{i_{n}}\left(T^{-n}\{V \in(x-\varepsilon, x+\varepsilon)\}\right)=1$. The mean value theorem entails $\lim _{n \rightarrow \infty} 2 \varepsilon h\left(\eta_{n}, i_{n}\right)=1$ for some $\eta_{n} \in(x-\varepsilon, x+\varepsilon)$ and since $\varepsilon$ was arbitrarily chosen, $h$ turns out to be unbounded.

\section{Applications}

We shall further consider two applications of Theorem 2 to supercritical branching processes. Let $\left\{Z_{n}: n \geqslant 0\right\}$ with $P\left(Z_{0}=i\right)>0$ for all $i \in N$ be a simple branching process and write $m=E\left(Z_{1}\right)$. It is known that if $1<m<\infty$ then there exists some norming constants $\left\{C_{n}\right\}$ such that $\lim _{n \rightarrow \infty} Z_{n} / C_{n}=W$ almost surely where $W$ is a proper random variable, $P(W=0)=q$ and $W$ admits an absolutely continuous distribution function on $(0, \infty)$ (see Seneta (1968) and Heyde (1970)). Besides $\lim _{n \rightarrow \infty} C_{n} / C_{n-1}=m$. It is easy to see that the conditions of Theorem 2 are satisfied with $\lambda_{0}=0$ and that $\left(\lambda_{1}, \alpha \lambda_{1}+\beta\right)$ can be taken to be $(1, m]$, whereas $\left(\lambda_{2}, \alpha \lambda_{2}+\beta\right)$ is absent since $P(W \geqslant 0)=1$. In fact, the result of Theorem 2 can be strengthened in this case to yield the following.

THEOREM 3. Let $\left\{Z_{n}: n \geqslant 0\right\}$ be a simple branching process with $1<m<\infty$. Then to any bounded harmonic function $h$ there corresponds a unique positive measure $\eta$ such that

$$
h(i)=q^{i}(0)+\int_{1}^{m} h(x, i) \eta(d x)
$$

where $q=\lim _{n \rightarrow \infty} P\left(Z_{n}=0\right)$.

Proof. If we notice that $P_{i}(W=0)=q^{i}$ then the representation (11) is a consequence of (6) and is thus valid for any harmonic function $h$ expressible as $h(i)=E_{i}(U)$ where $U$ is an invariant, bounded and $\sigma(W)$ measurable random variable. Further by a result established independently by Lootgieter (1977) and 
Brown (1977), $\mathscr{T}=\sigma(W)$ apart from null sets. Now, since any invariant variable is $\mathscr{T}$-measurable, an appeal to already mentioned Theorem 2 of Blackwell (1955) completes the proof.

Remark. Dubuc $(1970,1971)$ has shown that $\{h(x, i)\}$ are extremal harmonic functions provided that $E\left(Z^{2}\right)<\infty$ and if $E\left(Z \log ^{2} Z\right)<\infty$ any harmonic function (bounded or not) admits the representation (11). Pakes (1978), p. 70, has noticed that Dubuc's condition $E\left(Z \log ^{2} Z\right)<\infty$ can be relaxed to $E(Z \log Z)<\infty$. Lootgieter (1977) used a different method to show that if $1<m<\infty,\{h(x, i)\}$ are extremal harmonic functions, provided that they are finite. He has also given a representation for the space-time harmonic functions.

Our next application will refer to a regular branching process with infinite mean. In such a case it was proved in Schuh and Barbour (1977), Theorem 2.32, that there exists a slowly varying function $U$ and some norming constants $\left\{C_{n}\right\}$ with $\lim C_{n} / C_{n-1}=\alpha>1$ such that $\left\{U\left(Z_{n}\right) / C_{n}\right\}$ converges almost surely to a proper random variable $V=U(1 / T)$, where $P(T \geqslant x)=\exp (-x)$ for all $x>0$ and $P(V=0)=q$.

THEOREM 4. Suppose that $\left\{Z_{n}: n \geqslant 0\right\}$ is a regular branching process with infinite mean and assume that $U$ has a continuous derivative. Let $F$ be the distribution function of $V$ and $f$ its density function. Then

(a) for any $x>0$

$$
\begin{aligned}
& h(x, 0)=0 \\
& h(x, i)=\sum_{n=-\infty}^{\infty} \alpha^{n+1} i F^{i-1}\left(\alpha^{n+1} x\right) f\left(\alpha^{n+1} x\right) \text { for } i \geqslant 1
\end{aligned}
$$

is an harmonic function and for any fixed $i, h(x, i)$ is continuous;

(b) for any distinct values $y_{1}, \ldots, y_{m} \in(1, \alpha], h\left(y_{1}, i\right), \ldots, h\left(y_{m}, i\right)$ are linearly independent.

Proof. By equation (11) of Cohn (1977b) we get that

$$
F_{i}(x)=F^{i}(\alpha x) \text { for } i \geqslant 1
$$

and it is easy to see that $F_{0}(x)=1$ for $x \geqslant 0$. Elementary calculations lead us to (12). Given that $U$ is a slowly varying function and that $T$ is exponentially distributed, it is not difficult to convince ourselves that the series given by (12) converges uniformly in any closed interval $[a, b]$ with $a, b>0$ and thereofre $h(x, i)$ are continuous functions. 
To prove (b) we recall that in Remark after Theorem 2 we have noticed that there exists a sequence $\left\{i_{n}\right\}$ such that $\lim _{n \rightarrow \infty} P_{i_{n}}\left(T^{-n}\{V \in(x-\varepsilon, x+\varepsilon)\}\right)=1$ and that $\lim _{n \rightarrow \infty} 2 \varepsilon h\left(\eta_{n}, i_{n}\right)=1$ with $\eta_{n} \in((x-\varepsilon),(x+\varepsilon))$. Further we know that $i_{n} \in\left\{j:\left(U\left(Z_{n}\right) / C_{n}\right) \in(x-\varepsilon, x+\varepsilon)\right\}$ and therefore $i_{n} \in\left(U^{-1}\left(C_{n}(x-\varepsilon), U^{-1}\left(C_{n}(x+\varepsilon)\right)\right.\right.$. Elementary calculations can easily show that $h\left(x, i_{n}\right)$ is continuous in $x$, uniformly with respect to $n$, and this implies that $\lim _{n \rightarrow \infty} h\left(x, i_{n}\right)=\infty$. We take now $x=y_{k}$ where $k \in\{1, \ldots, m\}$. Since $\lim _{n \rightarrow \infty} P_{i_{n}}\left(\bigcup_{k=-\infty}^{\infty} T^{k}\{V \notin(x-\varepsilon, x+\varepsilon))=0\right.$ and because $(x-\varepsilon, x+\varepsilon)$ can be made small enough to ensure that all but one of the points $y_{1}, \ldots, y_{m}$ are outside $(x-\varepsilon, x+\varepsilon)$ we get that if $\lim _{n \rightarrow \infty} h\left(y_{k}, i_{n}\right)=\infty$ then $\lim _{n \rightarrow \infty} h\left(y_{k^{\prime}}, i_{n}\right)=0$ for $k^{\prime} \neq k$ and this entails the linear independence of

$$
h\left(y_{1}, i\right), \ldots, h\left(y_{m}, i\right) \text {. }
$$

\section{Acknowledgement}

The author is indebted to the referee for his useful comments on the manuscript.

\section{References}

D. Blackwell (1955), 'On transient Markov processes with a countable number of states and stationary transition probabilities', Ann. Math. Statist. 26, 654-658.

B. M. Brown (1977), 'The tail $\sigma$-field of a branching process' (unpublished).

H. Cohn (1977a), 'On the norming constants occurring in convergent Markov chains', Bull. Austral. Math. Soc. 17, 193-205.

H. Cohn (1977b), 'Almost sure convergence of branching processes', Z. Wahrscheinlichkeitstheorie und Verw. Gebiete 38, 73-81.

H. Cohn (1979), 'On the invariant events of a Markov chain', $Z$. Wahrscheinlichkeitstheorie und Verw. Gebiete 48, 81-96.

S. Dubuc (1970), 'La fonction de Greene d'un processus de Galton-Watson', Studia Math. 34, 69-87.

S. Dubuc (1971), 'Problèmes relatifs à l'itération des fonctions suggérés par les processus en cascade', Ann. Inst. Fourier, Grenoble, 21, 171-251.

C. C. Heyde (1970), 'Extension of a result by Seneta for the supercritical branching process', Ann. Math. Statist. 41, 739-742.

J. C. Lootgieter (1977), 'La $\sigma$-algèbre asymptotique d’une chaîne de Galton-Watson', Ann. Inst. Henri Poincaré, 13 (3), 193-230.

A. G. Pakes (1978), 'On the age distribution of a Markov chain', J. Appl. Probab. 15, 65-77.

F. Riesz and B. Sz.-Nagy (1955), Functional analysis (F. Ungar, New York).

D. Revuz (1975), Markov chains (North-Holland, Amsterdam).

E. Seneta (1968), 'On recent theorems concerning the supercritical Galton-Watson process', Ann. Math. Statist. 39, 2098-2102.

H.-J. Schuh and A. Barbour (1977), 'On the asymptotic behaviour of branching processes with infinite mean', Advances in Appl. Probability 9, 681-723.

Department of Statistics

University of Melbourne

Parkville, Australia 3052 